\title{
Verzögerte Kopfgeburt - Alternativen zum Kristeller-Handgriff
}

\author{
Ulrike Harder, Hebamme, Lehrerin für Hebammenwesen, freie Dozentin, Berlin
}

\section{Zur Beschleunigung einer verzögerten Kopfgeburt eignen sich viele verschiedene Maß- nahmen. Die Anwendung des Kristeller-Handgriffs sollte vermieden werden, da der äußere Fundus-Druck meist schmerzhaft ist und bis dato in keiner Studie einen Nutzen für Mutter und Kind zeigte.}

Tritt in der aktiven Austreibungsphase (AP) der auf Beckenboden stehende oder bereits sichtbare Kopf nur sehr langsam bis gar nicht tiefer oder bleibt seine Deflexions-Bewegung aus, ist je nach Geburtssituation und Geduld der Beteiligten eine Unterstützung angezeigt.

\section{Dauer der Austreibungsphase}

Es gibt keine allgemein anerkannte Definition einer regelrechten Geburtsdauer. Da die Zeitangaben in der Fachliteratur variieren, muss in der letzten Geburtsphase keine unnötige Hetze gemacht werden. Lehrbuch-Angaben zur Definition der protrahierten Austreibungsphase (ab 2 Stunden bei Erstgebärenden, ab 1 Stunde bei Mehrgebärenden) verunsichern, obwohl immer darauf hingewiesen wird, dass bei Überschreitung der Zeit die Ursache evaluiert und therapiert werden sollte, was aber nicht gleichbedeutend mit einer operativen Geburtsbeendigung ist [1].

Das American College of Obstetricians and Gynecologists hat 2014 in seinem Consensus Papier zur Senkung der Kaiserschnittrate längere, evidenzbasierte Zeiten zur Dauer der AP definiert [2, 3]. Nachdem in mehreren Studien bei Erstgebärenden mit bis zu 3-5 Stunden AP keine gravierenden Probleme bei den Kindern festgestellt wurden und eine
Studie bei Mehrgebärenden mit mehr als 3 Stunden AP niedrigere Apgar-Werte und höhere Verlegungsraten fand, wurden folgende Zeiten festgelegt:

Für die Austreibungsphase ist eine längere Geburtsdauer tolerierbar, wenn die mütterliche und kindliche Kondition es zulässt [2].

- Multipara: 2 Std., mit PDA 3 Std.

- Erstpara: 3 Std., mit PDA 4 Std.

- längere Zeiten sind möglich, wenn z. B. bei kindlichen Fehleinstellungen ein Geburtsfortschritt dokumentiert werden kann.

Dies bedeutet selbstverständlich nicht, dass wir in Zukunft immer nur abwarten bis die maximale Zeit erreicht ist. Wird ein langsam oder gar nicht tiefer tretender Kopf diagnostiziert oder soll die Geburt aufgrund eines pathologischen CTGs beschleunigt werden, sind geburtsfördernde Maßnahmen zu ergreifen, um die Geburt für Mutter und Kind schonend zu Ende zu bringen. Das zu frühe aktive Mitpressen und ein hochdosierter Oxytocin-Tropf gehören allerdings nicht dazu, denn beide Maßnahmen verschlechtern die uterine Durchblutung und verursachen oft ein pathologisches CTG-Muster. Die Geburtshelfer geraten dann in Zugzwang und „müssen“ mit einer Sectio, dem Kristeller-Handgriff, dem Forzeps oder der Vacuumextraktion die Geburt beenden.
Geduld ist ein wichtiger Geburtshelfer. In der Austreibungsphase sollte die Frau erst dann zum aktiven Mitschieben aufgefordert werden, wenn der Kopf in der Scheide sichtbar wird (Erstpara) oder auf Beckenboden steht (Multipara).

Diese alte Regel hat sich gut bewährt, damit die empfohlene aktive Pressphase von 20-30 Minuten nicht überschritten wird $[1,4,5]$. Um die passende Therapie zu finden sollte zunächst die Ursache der Verzögerung abgeklärt werden.

\section{Ursachen für verzögerte Kopfgeburt}

- Wehenschwäche und Erschöpfung

- Mitdrücken der Frau ist nicht effektiv:

- kein Halt für ihre Hände und Füße

- Anleitung zum Mitschieben nicht umsetzbar

- Vorwölbung einer breiten Rektusdiastase

- PDA-Wirkung zu stark

- Gebärposition energetisch ungünstig

- Seelische Blockaden

- Rigider Beckenboden

- Kopf noch nicht ausrotiert

- Kopf-Becken-Missverhältnis,

Fehleinstellungen 


\section{Therapie-Wahl}

Je nach Ursache und geburtshilflicher Situation lässt sich der Kopfaustritt durch folgende Maßnahmen fördern und beschleunigen.

\section{Wehenschwäche/Erschöpfung}

Ein Geburtsstillstand in der Durchtrittsphase kann toleriert werden, wenn es dem Kind gut geht. Die Wehen werden wieder angeregt durch leichte Bauchmassage mit Uterustonikum-Öl, einen Wechsel der Gebärposition (Vierfüßler, Hocker, tiefe Hocke, Seitenlage) oder durch eine Oxytocininfusion. Ist die Mutter sehr gestresst und erschöpft und hat lange keine Nahrung zu sich genommen, sind ihre Oxytocin-Rezeptoren im Myometrium nur schwer zu stimulieren. Ein rasch einlaufender „Nährtropf“ (z.B. Sterofundin ${ }^{\circledR}$ ) kann dann wirkungsvoller sein als ein Oxytocintropf.

\section{Mitdrücken nicht effektiv}

Die Gebärende braucht beim Mitdrücken etwas zum Festhalten für ihre Hände, um den Oberkörper zu stabilisieren, z. B. Griffe am Bett (Abb.1), Hände des Part-

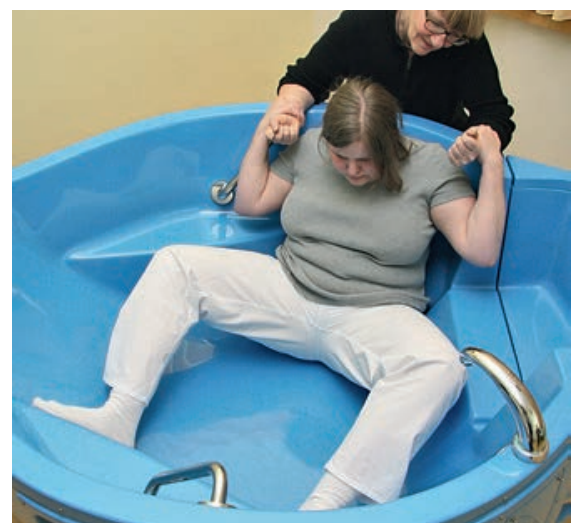

Abb. 3 Einigen Frauen fehlt in der großen Gebärwanne der Halt. Bitten wir sie zum Mitschieben quer in die Wanne, kann sie sich hinten mit dem Oberkörper und vorne mit den Füßen am Wannenrand anstemmen. Da sie jetzt die Haltegriffe der Wanne nicht nutzen kann, hält sie sich an den Unterarmen der Begleitperson fest. ners (Abb. 2,3) oder ein Tuch zum Ziehen (Abb.4). Dieser Halt entlastet ihr Becken und macht es mobiler, außerdem wird so ihr Beckenboden weicher. Kann die Frau ihre Füße bzw. Fersen beim Mitschieben gegen eine feste Unterlage drücken, gelingt ihr eine deutlich intensivere, kraftvollere Bauchpresse [6, 7]. Wenn die Hebamme der Gebärenden dieses bereits in der Eröffnungsphase erklärt und verschiedene Position zum Herausschieben des Kindes mit ihr probiert, weiß die Frau in der letzten Geburtsphase, worauf

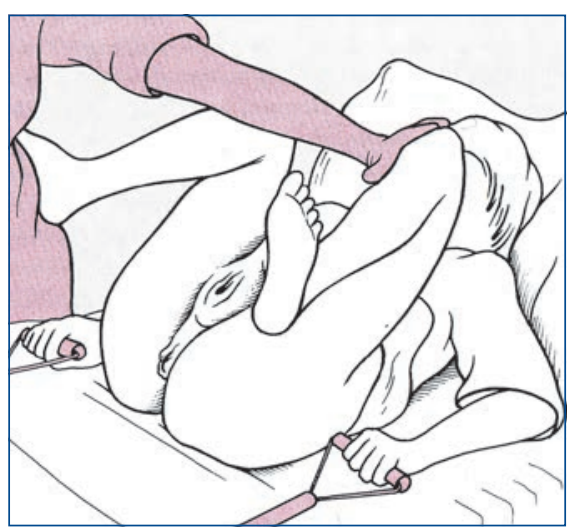

Abb. 1 Dieses Bild mit Haltegriffen für die Geburt in Rückenlage befindet sich im Hebammenlehrbuch (Hrsg. Gerhard Martius) von der 1. Auflage (1962) bis zur 7. Auflage (1997). Leider wurden die Griffe nur bis in die 1970-er Jahre verwendet. (c) Hebammenlehrbuch [4]

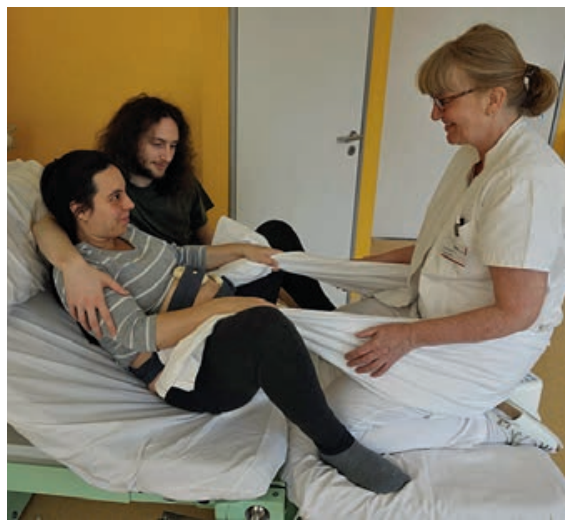

Abb. 4 Die Hebamme kniet auf einem festen Kissen vor der Frau im Gebärbett und reicht ihr ein Laken/Tuch zum Festhalten. Kann die Frau mit den Händen in die gleiche Richtung ziehen, in die sie das Kind schiebt, wird ihr das Mitdrücken sehr erleichtert. es ankommt und findet leichter den passenden Halt für Hände und Füße.

\section{Anleitungen nicht umsetzbar}

Deutliche Atemkommandos der Geburtshelfer „Wehe da? Gut jetzt tief Luft holen, Luft anhalten und drücken, drücken, drücken..." passen oft nicht zu dem persönlichen Atemmuster der Frau und sie kann in dem vorgegebenen Rhythmus kaum Druck nach unten aufbauen. Wird

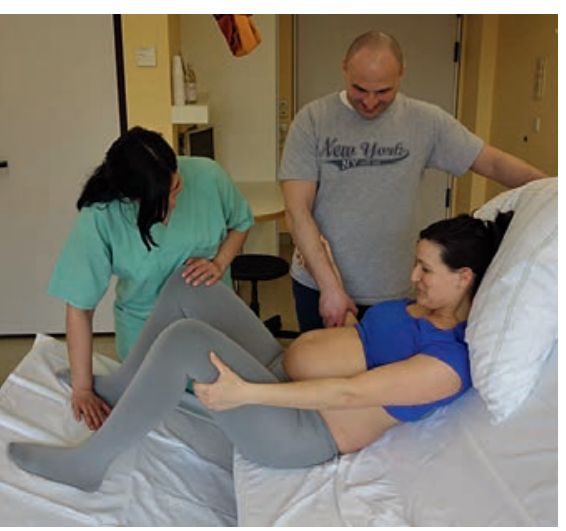

Abb. 2 Zum energetischen Mitdrücken zieht die Frau mit einer oder beiden Händen ihren Partner zu sich ins Bett. Ihre Füße kann sie gut anstemmen, wenn sich (wie hier) das Fußteil des Bettes schräg stellen lässt. Alle Fotos: Ulrike Harder und Hebammen/Schülerinnen aus Berlin

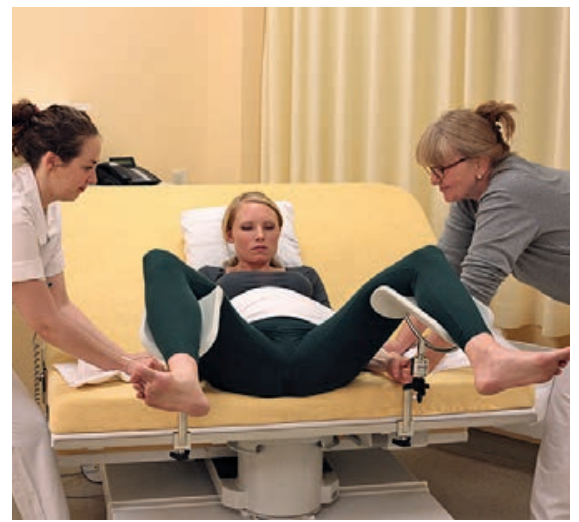

Abb. 5 Während der Wehe kann die Bauchpresse mit einem über den Bauch gespannten Laken unterstützt werden (z. B. bei großer Rectusdiastase). 


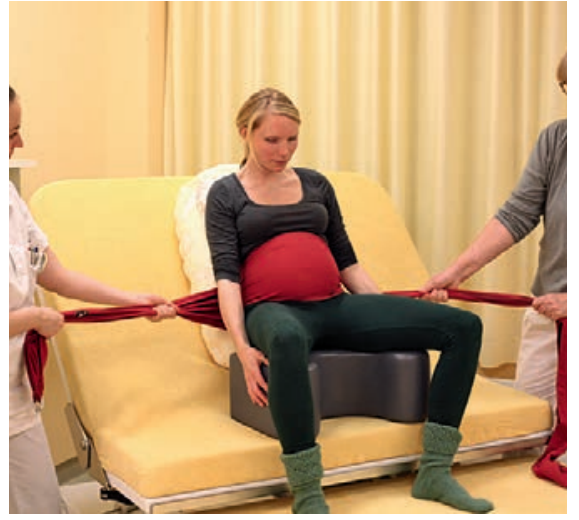

Abb. 6 Ein Tragetuch (mexikanisch: Rebozo) wird über die oberen 2/3 des Bauches gelegt und hinten am Rücken der Frau gekreuzt. So kann es während der Wehe zur Verstärkung der Bauchmuskeln langsam zugezogen werden. Die Anwendung ist auf dem Gebärhocker, im Bett und in Vierfüßler-Position möglich.

die Gebärende hingegen aufgefordert, bei jeder Wehe nach eigenem Gefühl kräftig mitzuschieben, findet die Frau meist binnen weniger Wehen ihren Rhythmus, um das Kind herauszudrücken. Oft wird sie dann in der Wehe dreibis fünfmal mittdrücken und dabei etwas ausatmen (knurren, schreien etc.), was aber meist nicht hinderlich ist [8].

Nach einem Literaturreview (10 Studien) scheint angeleitetes Mitdrücken in der AP gegenüber selbstbestimmtem Schieben/Drücken keine Vorteile hinsichtlich Dauer der AP, Geburtsmodus, Apgar-Werte und Nabelschnur$\mathrm{pH}$ zu haben [9]. Durch forciertes Mitdrücken erhöht sich das Risiko für maternale und fetale Hypoxie [10].

\section{Breite Rektusdiastase}

Wölbt sich beim Mitdrücken der Frau ein breiter Wulst zwischen den geraden Bauchmuskeln hervor, kann sie nur wenig Druck nach unten aufbauen. Ein über den Bauch der Frau gelegtes und in der Wehe zugezogenes Tuch (Abb. 5,6) kann ihre Bauchmuskeln stabilisieren und den intrauterinen Druck nach unten verstär-

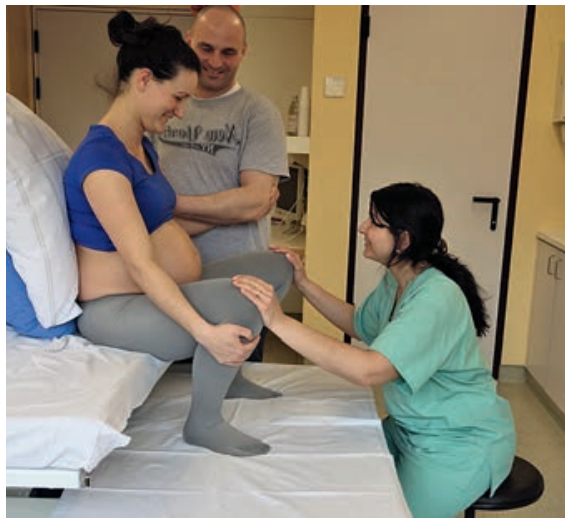

Abb. 7 Der Kniedruck erfolgt von vorne, die Beine werden in Richtung des Beckens geschoben. Dadurch wird das Becken etwas aufgerichtet, was das Mitschieben in der Wehe sehr erleichtert. CAVE: nicht die Beine auseinanderdrücken!

ken. In Rückenlage sind auch flach aufgelegte Hände hilfreich (s.u. KristellerHandgriff).

\section{PDA-Wirkung}

Hat die Frau wegen einer Periduralanästhesie kein Gefühl für die Druckrichtung (fehlender Pressreflex) kann eine warme Kompresse am Damm hilfreich sein. Einige Hebammen drücken auch mit 2 Fingern in der Scheide auf das Rektum, um den Pressreflex auszulösen (Nutzen ist nicht erwiesen). Besonders Gebärende mit PDA werden in aufrechter Position und mit Halt an Händen und Füßen den erforderlichen Druck besser aufbauen können als im Liegen. Moderne Anästhesieverfahren (Mobil-PDA) machen es möglich, dass auch diese Frauen sich in der AP bewegen und auf dem Hocker, in Vierfüßler-Position oder im Stehen gebären. Dies gilt es zu nutzen.

\section{Ungünstige Gebärposition}

Die halbsitzende Rückenlage ist eine energetisch schwierige Position zum Herausdrücken des Kindes [11], besonders

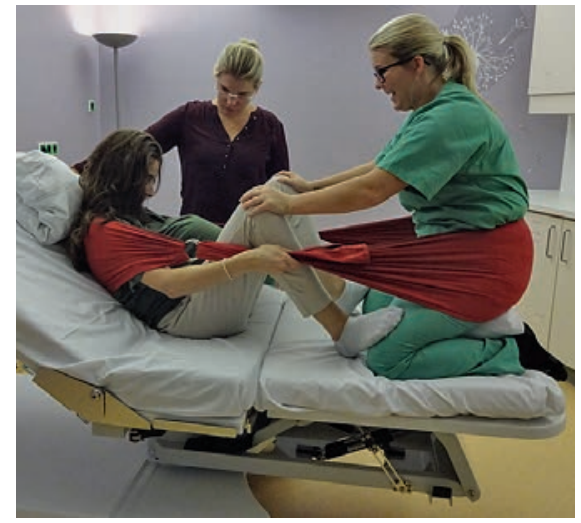

Abb. 8 Kniedruck während der Wehe. Frau und Hebamme befinden sich in einer Ring-Schlaufe nach Harder. Diese gibt der Frau Halt im Rücken und ihre Hände können am Tuch ziehen. Legt sich die Hebamme die Schlaufe um das Gesäß, wird sie dem Zug gut standhalten. Beginnt der Kopf auszutreten, beendet sie sofort den Kniedruck und hat ihre Hände frei für die Kindsentwicklung.

wenn die Frau aufgefordert wird, mit den Händen in den Kniekehlen ihre Beine hochzuhalten. Das Festhalten der eigenen Beine ist kraftraubend, gibt der Frau keinen Halt und ihre Bauchmuskeln lassen sich in der gekrümmten Haltung mühsamer anspannen als bei geradem Rücken [12]. Auch in Rückenlage brauchen die Füße Gegendruck im Bett. Zusätzlich kann ein zum Körper der Frau gerichteter Kniedruck ihr das Mitschieben erleichtern, da er das Becken etwas aufrichtet (Abb. 7,8). Die Gebärende kann auch im Bett aus der halbsitzenden Position für einige Wehen zum Mitschieben in die tiefe Hocke gebracht werden (Abb.9,10). Ebenso hilfreich sind ein Wechsel auf den Gebärhocker, asymmetrische Positionen (Abb.11,12) und besonders bei dorso posterioren Einstellungen die Vierfüßler-Position (Abb. 13, 14).

\section{Seelische Blockaden}

Die Frau befragen ob sie etwas (oder uns) als störend empfindet, ob die Geburt bei ihr unangenehme Erinnerungen aktiviert und ob wir etwas tun oder unterlassen können, um ihr die Situation und 
Geburtsposition zu erleichtern. Oft kann eine seelische Ursache (z.B. Traumatisierung) im Routine-Alltag des Kreißsaales nicht genau ergründet werden. In jedem Fall hilfreich sind: Respektvoller Umgang mit der Frau, Wahrung ihrer Intimsphäre (z.B. Beine nicht auseinanderdrücken), ruhige Erklärung aller Maßnahmen und wenn es dem Kind gut geht, die Akzeptanz der langsamen Kopfgeburt. Keine

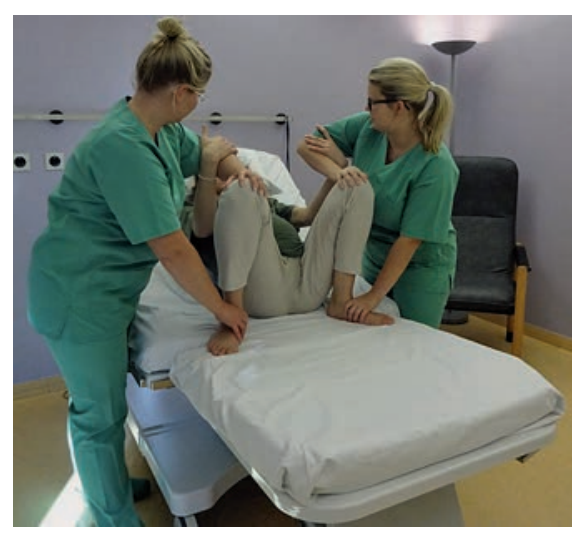

Abb. 9 Zur Wehe wird die Frau im Bett zum Mitschieben in die tiefe Hocke gebracht, in der Wehenpause kann sie sich im Liegen ausruhen. Die Helferinnen stellen mit einer Hand den Fuß eng an den Körper und legen die andere Hand auf das Knie. Dann bitten sie die Frau, sich an ihren Armen festzuhalten (weiter s. Abb. 10).

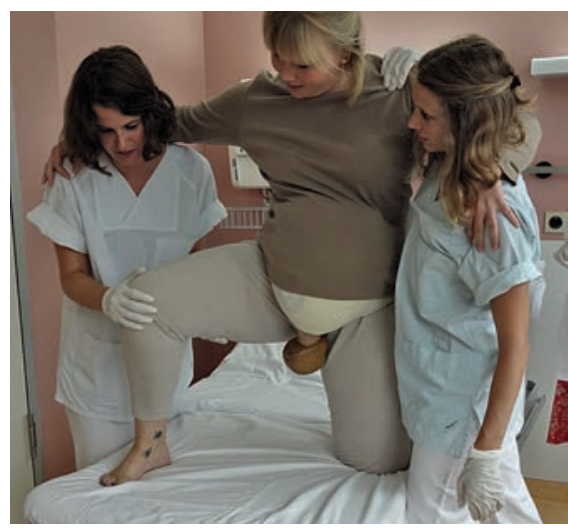

Abb. 12 Bei asymmetrischem Knien (Hirtenstellung) wird das Becken etwas schräg gestellt und das Kind bekommt z. B. bei aufgestelltem rechten Bein rechts mehr Platz für den Kopfdurchtritt. Außerdem kann die Frau beim Mitschieben einen Fuß in das Bett drücken. CAVE: Geburt erfolgt eventuell sehr schnell in dieser Position.
Hektik verbreiten, manche Kinder brauchen etwas länger.

\section{Rigider Beckenboden}

Die Verspannung wird günstiger weise bereits zu Beginn der AP bei einer vaginalen Untersuchung erkannt und früh therapiert. Zur Lockerung der Beckenund Beckenboden-Muskulatur haben

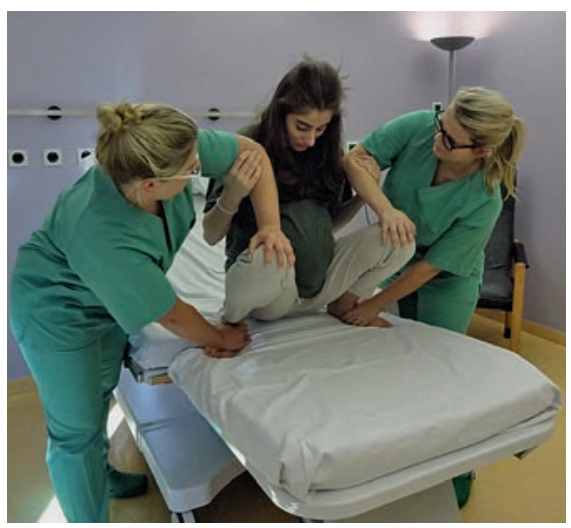

Abb. 10 Durch Druck auf die Knie wird nun die Frau recht leicht in die Hocke gebracht. Befindet sich ein Seil/Tuch an der Decke über dem Bett oder wird eine Ring-Schlaufe am Fußende des Bettes befestigt, kann sich die Frau am Tuch (statt an den Helferinnen) hochziehen und beim Mitschieben sehr gut festhalten.

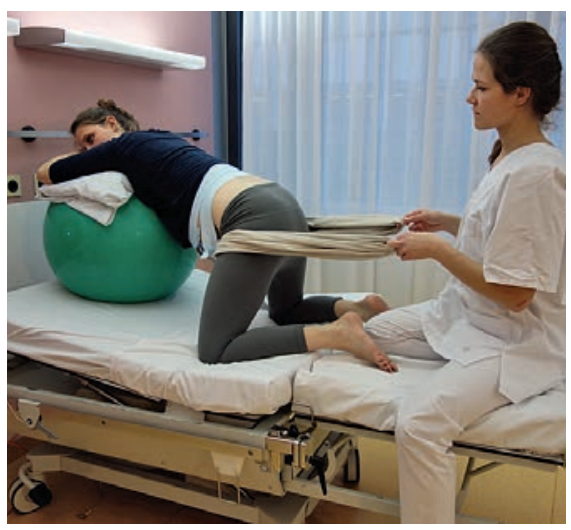

Abb. 13 In der Wehenpause sollte sich die Frau auch in Vierfüßler-Position gut ausruhen können. Auf dem Ball wird ihr Oberkörper gut gelagert. Die Hebamme kann zusätzlich versuchen, die Anspannungen im Becken durch ein leichtes Schütteln zu lockern. Hierfür eignet sich ein über die Leisten gelegtes Tuch (Rebozo), welches leicht hin und her bewegt wird. sich die Seiten-Bauch-Lage (Abb. 15), das Rollen in der Ring-Schlaufe (Abb. 15, 16) und Positionen, in denen der Beckenboden frei hängen kann, bewährt (z.B. tiefe Hocke am Tuch oder auf dem Schoß von Helferinnen/Partner). Wird der sichtbare Kopf über mehrere Wehen von dem sich nicht dehnenden Gewebe im Beckenausgang zurückgehalten, kann zur Erweiterung eine Episiotomie nötig sein.

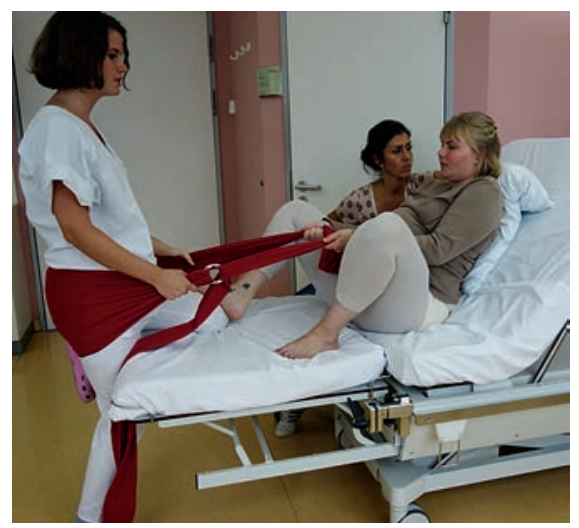

Abb. 11 Steht die Hebamme mit einem Bein vor und mit einem Knie im Bett, kann die Gebärende einen Fuß auf ihren Oberschenkel stellen und so in asymmetrischer Haltung mitdrücken. Eine passend eingestellte Ring-Schlaufe (oder ein Bettlaken) um das Gesäß der Hebamme ermöglichen hier ein Ziehen und Mitschieben in die gleiche Richtung.

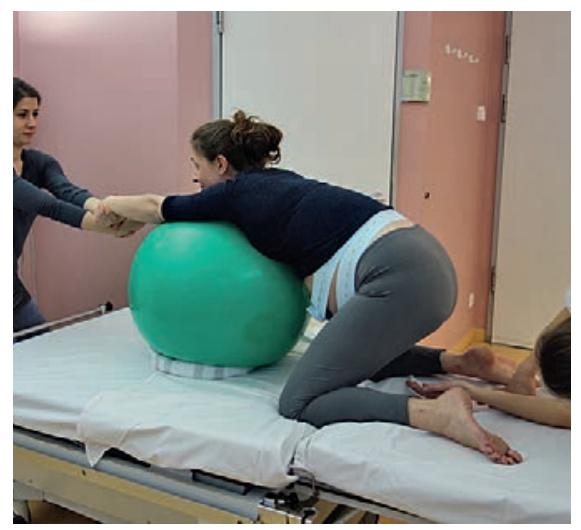

Abb. 14 Zum Mitschieben in VierfüßlerPosition wird das Bett etwas von der Wand zurückgezogen. Die Begleitperson stellt sich hinter das Kopfteil des Bettes, damit die Frau an ihren Armen ziehen kann, um besser mitdrücken zu können. Damit der Ball nicht vom Bett rollt, liegt er auf einem zum Ring geformten Handtuch. 


\section{Kopf nicht ausrotiert}

Solange die Pfeilnaht nicht gerade steht, gestaltet sich die Kopfgeburt schwierig. Wechselnde Seiten-Bauchlagerung (Simslage) mit stark angewinkeltem oberen Bein (Abb. 17), leichtes Mitdrücken in der Wehe oder Beckenmobilisation in Seitenlage (Abb. 18, 19) unterstützen die Kopfdrehung [13]. Die Mobilisation wird während mehrerer Wehen erst auf der Seite des kindlichen Rückens, dann auf der anderen Seite ausgeführt. Wenn nötig muss der Kopf mit der Saugglocke gedreht und extrahiert werden.

\section{Kopf-Becken-Missverhältnis}

Passt der Kopf wegen einer Einstellungs-/ Haltungsanomalie oder einem engen Schambogenwinkel, vorspringenden Spinae oder störendem Steißbein nur knapp durch Beckenmitte und Beckenausgang, kann mit dem äußeren Beckendruck das Becken etwas geweitet werden (Abb. 20, 21,22). Der Druck erfolgt während der Wehe, damit der nach unten drängende Kopf die veränderten Beckenräume zum Tiefertreten nutzen kann [14, 15]. Auch die wechselseitige Beckenmobilisation in Seitenlage (s.o.) und Vierfüßler-Positionen sind hilfreich.

\section{Kristeller-Handgriff}

\section{Studienlage}

Der seit 150 Jahren angewandte Kristeller-Handgriff ist wenig erforscht und laut Cochrane Database of Systematic Review [16] liegen keine richtigen Evidenzen für den Fundus-Druck vor. Ob durch FundusDruck die Geburtsdauer verkürzt und das fetale Outcome verbessert werden kann, konnten etliche Studien nicht belegen $[17,18,19]$ außerdem wird regelmäßig über Komplikationen durch die Anwendung berichtet [20, 21, 22].

Die Anwendung des Kristeller-Handgriffs (Uterine Fundal Pressure) wird kontrovers diskutiert. Die Beschreibungen di-

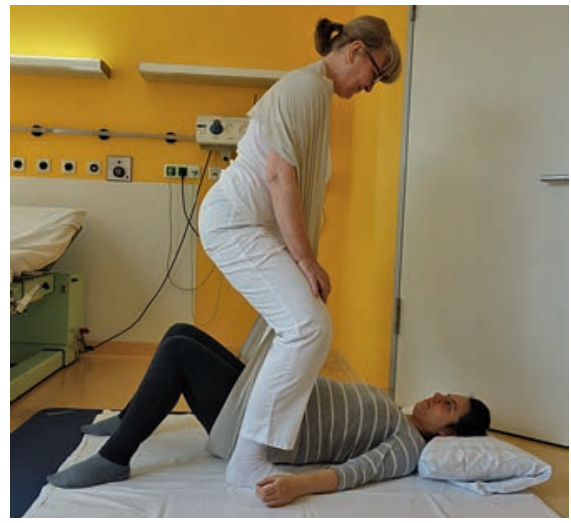

Abb. 15 Das Schaukeln und Rollen des Beckens in einem Tuch (hier Ring-Schlaufe nach Harder) lockert Rücken, Iliosakralbereich und den Beckenboden. Die meisten Frauen empfinden das als sehr wohltuend und lassen sich gerne über mehrere Wehen-Pausen (einige auch während der Wehe) hin und her rollen. Zum Anheben muss die Hebamme ihren Rücken ganz gerade halten und die Knie strecken (weiter s. Abb. 16).

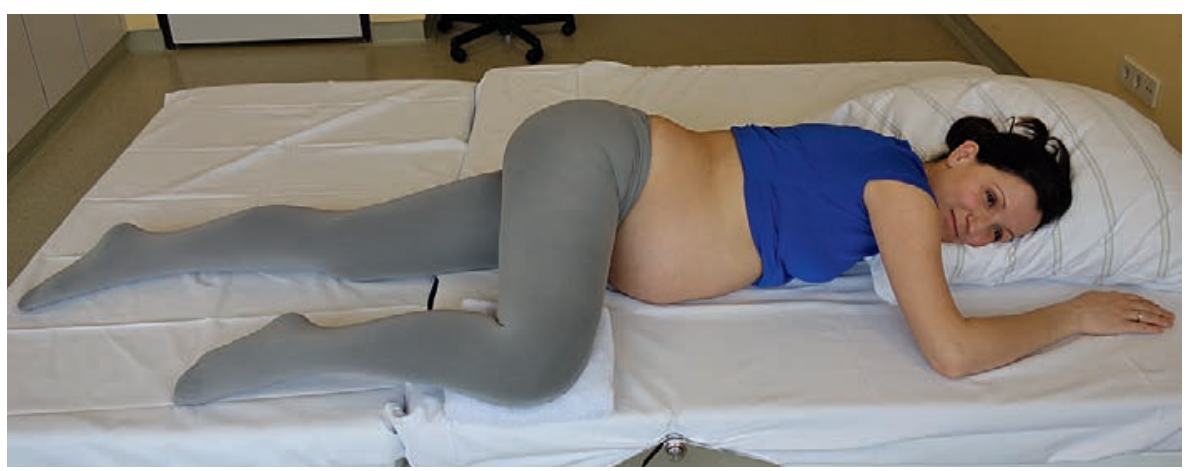

Abb. 17 Seiten-Bauchlage (Simslage) auf der linken Seite. Das stark angewinkelte rechte Bein und der vom Bett etwas angehobene Bauch schaffen dem Kind auf der rechten Seite des Beckens mehr Platz zur Rotation.

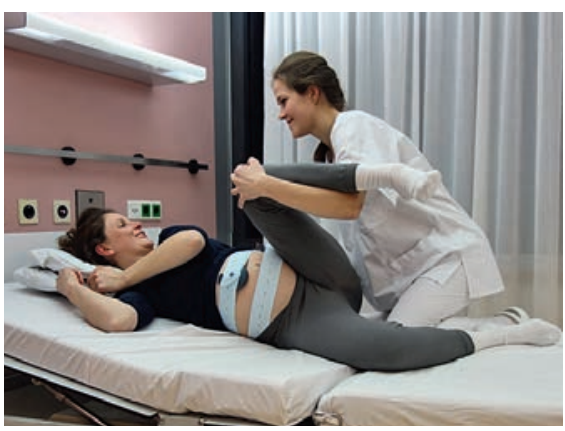

Abb. 18 Beckenmobilisation Seitenlage während der Wehe zur Erweiterung des Beckenausgangs: Die Hebamme kniet mit einem Bein im Bett, sie legt sich Unterschenkel und Knie der Frau auf den Arm und drückt ihr dieses an den Körper - optimal bei der Ausatmung (weiter s. Abb. 19).

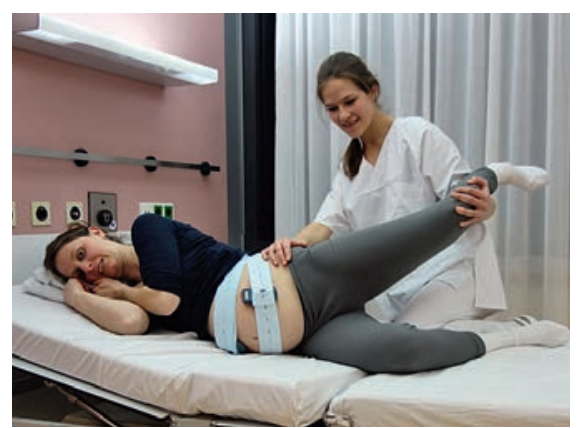

Abb. 19 Anschließend wird das Bein zur Erweiterung des Beckeneingangs nach hinten geleitet, wobei die Hebamme das Becken mit der anderen Hand leicht nach vorne rollt (optimal bei der Einatmung). In der Wehenpause legt oder stellt die Hebamme das Bein ab. 


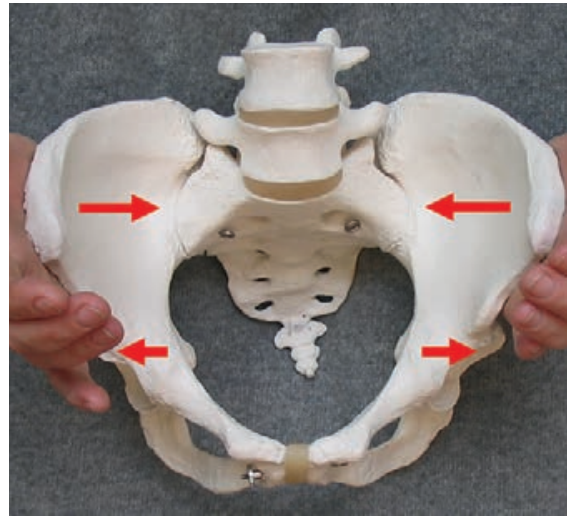

Abb. 20 Äußerer Beckendruck zur Erweiterung des Beckenausgangs: Die Hände liegen auf den Darmbeinschaufeln (nicht auf dem Darmbeinkamm) und drücken in der Wehe das Becken zusammen, damit die Spinae ischiadica für den Kopfaustritt etwas auseinander weichen können.

vergieren in der Fachliteratur bezüglich der Indikationen, Kontraindikationen und empfohlenen Technik. Der Druck auf den Fundus erfolgt immer langsam ansteigend und wehensynchron. Die Technik kann mit zwei flach aufgelegten Händen [4, 23] (Abb. 23), oder mit einer Hand $[5,24,25]$ (Abb. 24) oder mit dem Unterarm $[26,27]$ durchgeführt werden.

Der oft zu sehende Fundus-Druck mit dem Unterarm (Abb. 25) ist nicht zu empfehlen, da sich das Kind kaum in Führungslinie halten lässt und der Druck der harten Unterarmknochen Gewebeschädigungen am Myometrium und Hämatome verursachen kann (Abb.26). Auch wenn sich der Geburtshelfer auf der gegenüberliegenden Seite an Betttuch oder Stecklaken festhält, besteht immer die Gefahr, dass der Ellenbogen seitlich schmerzhaft auf die Rippen drückt oder bei Abrutschen eine Rippenfraktur verursacht.

\section{Fazit}

Mit etwas mehr Geduld in der letzten Geburtsphase, wechselnden Gebärpositionen, spätem aktiven Mitschieben und speziellen Beckenbewegungen erübrigt sich der Einsatz des schmerzhaften Fun-



Abb. 21 Äußerer Beckendruck in Seitenlage: Dazu bekommt die Frau ein Kissen zwischen die angewinkelten Beine. Nach dem Ertasten der Beckenschaufel erfolgt der Druck wehensynchron mit beiden Händen leicht schräg nach vorne. Der Druck darf keine Schmerzen verursachen!

dus-Drucks. Wenn wir der Frau eine energetische Position mit Halt für Arme und Füße ermöglichen, wird sie ihr Kind meist selbst herausdrücken können ohne Kristeller-Hilfe.

\section{Literatur}

1 Drack G, Schneider H. Pathologische Geburt. In: Schneider/Husslein/ Schneider, Hrsg. Die Geburtshilfe. 4. Aufl. Heidelberg: Springer 2013

2 ACOG Obstetric Care Consensus No.1 Safe prevention of the primary cesarean delivery. Obstet Gynecol 2014; 123: 693-711

3 Schwarz C. Dem Wahnsinn Einhalt gebieten? Die aktuelle US-amerikanische Leitlinie zur Senkung der Kaiserschnittrate. Die Hebamme 2014; 27 : 84-90

4 Martius G, Heidenreich W. Hebammenlehrbuch. 7. Aufl. Stuttgart: Thieme 1997

5 Dudenhausen JW, Hrsg. Praktische Geburtshilfe mit geburtshilflichen Operationen. 21. Aufl. Berlin: De Gruyter 2011

6 Heller A. Schieben - ein natürliches und schonendes Gebärverhalten. Die Hebamme 2004; 17: 22-31

7 Fischer H (2007). Atlas der Gebärhaltungen. 2. Aufl. Stuttgart: Hippokrates 2007

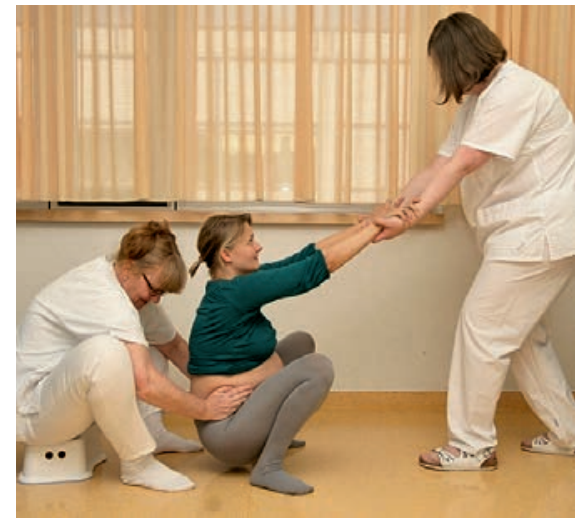

Abb. 22 Äußerer Beckendruck in tiefer Hocke: Wenn die Hebamme auf einem Hocker hinter der Frau sitzt, kann sie mit ihren Beinen die Kraft der Arme beim Drücken unterstützen.

8 Roberts JE et al. A descriptive analysis of involuntary bearing down efforts during the expulsive phase of labour. J Obstet Gynecol Neonatal Nurs 1087; 16: 48-55

9 Bosomworth A, Bettany-Saltikov J. Just take a deep breath ... A reb'view to compare the effects of spontaneous versus directed Vasalva pushing in the second stage of labour on maternal and fetal wellbeeing. MIDRIS 2006; 16 : 157-165

10 Beckermann MJ, Perl FM. Frauen-Heilkunde und Geburts-Hilfe, Integration von Evidence-BasesMedicine. Basel: Schwabe 2004

11 Kuntner L. Die Gebärhaltung der Frau. 4. Aufl. München: Marseille 1994 (jetzt als DVD erhältlich beim Deutschen Hebammen Verband)

12 Franke T. In: Schwarz C, Stahl K (Hrsg). Geburt in Bewegung - die Kräfte nutzen. Hannover: Staude 2015

13 Olshausen A. Fehlendes Tiefertreten des Köpfchens. In: DHV (Hrsg.) Geburtsarbeit. 2. Aufl. Stuttgart: Hippokrates 2013

14 Simkin P, Ancheta R. Schwierige Geburten - Leicht gemacht. 2. Aufl. Bern: Huber 2005

15 Harder U. Einstellungs- und Haltungsanomalien. In: Stiefel A, Geist C, Harder U. (Hrsg.) Hebammenkunde 5. Aufl. Stuttgart: Thieme 2013 


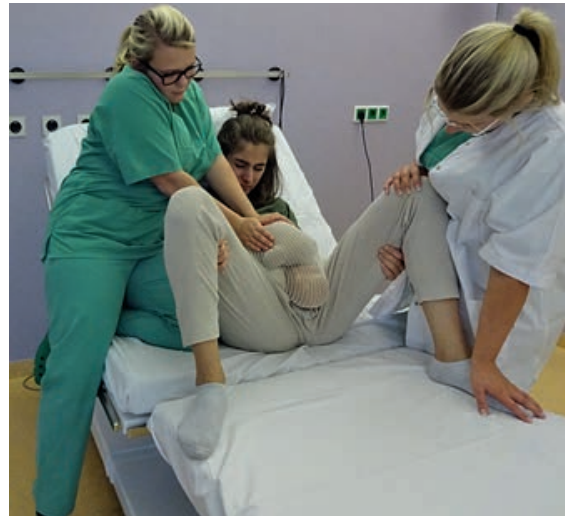

Abb. 23 Kristeller-Handgriff mit flach aufgelegten Händen: Nach dem Abtasten des Fundus wird das Kind in Führungslinie gebracht und dann wehensynchron Richtung Beckenausgang geschoben. Die Hebamme kniet mit einem Bein im Bett, damit sie sich hinten am Bettoberteil mit dem Gesäß abstützen kann. So bekommt sie genügend Kraft zum Mitschieben.



Abb. 26 Sichtbare Hautverletzung nach Anwendung des Kristeller-Handgriffes mit dem Unterarm am 6. Tag post partum. Foto: privat

16 Verheijen EC et al. Fundal pressure during the second stage of labour. Cochrane Database of Systematic Review 2009, Issue 4. Art. No.: CD006067

17 Api $\mathrm{O}$ et al. The effect of uterine fundal pressure on the duration of the second stage of labor: A randomized controlled trial. Acta Obsterica et Gynecologica Scandinavica 2009; 88: 320-324

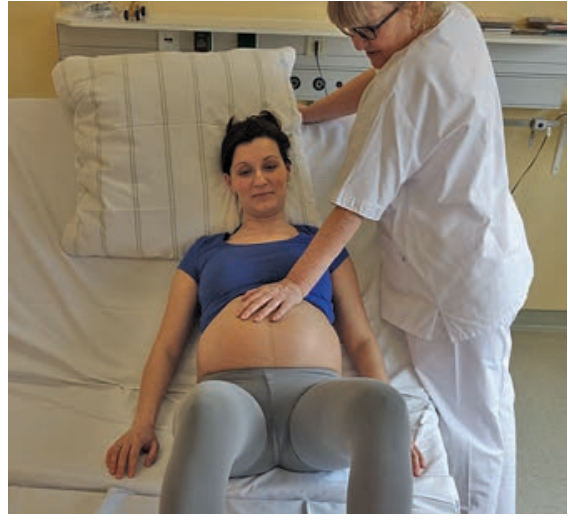

Abb. 24 Kristeller-Handgriff mit einer Hand, wenn der Kopf noch nicht ausrotiert ist: Der Druck erfolgt von der Seite der kleinen Teile, um Beugung und Drehung des Kopfes zu fördern. Stützt sich die Geburtshelferin mit einer Hand am Bettoberteil ab, hat sie mehr Kraft, um das Kind mit der anderen Hand zum Beckenausgang zu schieben.

18 Buhimschi et al. The effect of fundal pressure manoeuvre on intrauterine pressure in the second stage of labour. International Journal of Obstetrics and Gynaecology 2002; 109: 520-526

19 Schulz-Lohmeyer et al. Die KristellerTechnik: Eine prospektive Untersuchung. Geburtsh u Frauenheilkunde 1999; 59: 558-561

20 Falkert A. Komplikationen bei Kristeller Handgriff und vaginal operativer Entbindung. Pädiatrische Praxis 2011; 77: 243-244

21 Mahendru R et al. Age old practice of Uterine Fundal Pressure in Labour more risky than beneficial. Biomedical Reseach 2010; 21: 295-296

22 Hasegawa et al. Uterine rupture after the uterine fundal pressure maneuver. J Perinat Med 2015; 43 (6): 785-788

23 Breckwold M. Gynäkologie und Geburtshilfe. Stuttgart: Thieme 2008

24 Kiechle M. Gynäkologie und Geburtshilfe. 2. Aufl. München: Urban \& Fischer in Elsevier 2011

25 Harder U. Verzögerte und forcierte Kopfentwicklung. In: Stiefel A, Geist C, Harder U. (Hrsg.) Hebammenkunde 5. Aufl. Stuttgart: Thieme 2013

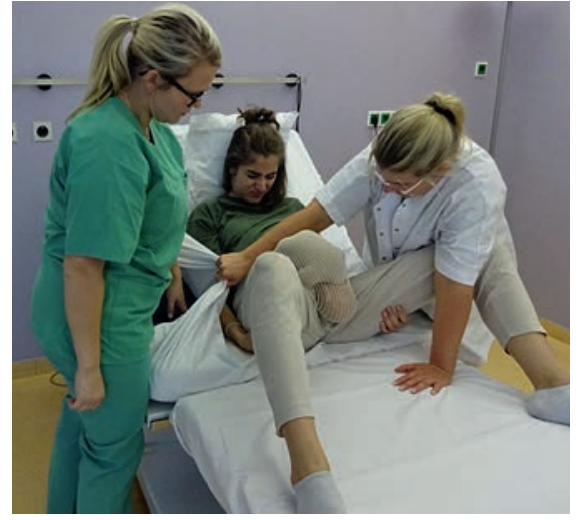

Abb. 25 Der Kristeller-Handgriff mit dem Unterarm ist für die Frau sehr schmerzhaft und birgt diverse Verletzungsrisiken (Gewebeschädigungen, Rippenfraktur, Plazentaablösung etc.). Auch kann das Kind beim Drücken nur schlecht in Führungslinie gehalten werden.

26 Bühling K. Intensivkurs Gynäkologie und Geburtshilfe. München: Elsevier 2009

27 Kainer F. Kristellerhilfe - obsolet der sinnvoll? Die Hebamme 2016; 20: 238-240

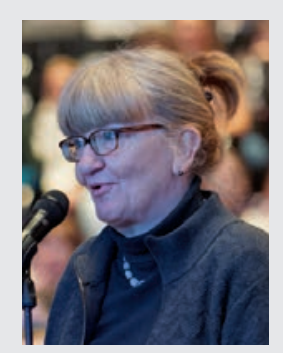

Ulrike Harder

Hebamme, Lehrerin für

Hebammenwesen, freie Dozentin,

Berlin

u.harder@gmx.de 